\title{
Effect of carvedilol on silent anthracycline-induced cardiotoxicity assessed by strain imaging: A prospective randomized controlled study with six-month follow-up
}

\author{
Ali Elitok ${ }^{1}$, Fahrettin $\mathrm{Oz}^{1}$, Ahmet Y. Cizgici ${ }^{1}$, Leyla Kilic ${ }^{2}$, Rumeysa Ciftci ${ }^{2}$, \\ Fatma Sen ${ }^{2}$, Zehra Bugra $^{1}$, Fehmi Mercanoglu ${ }^{1}$, Aytac Oncul ${ }^{1}$, Huseyin Oflaz ${ }^{1}$ \\ ${ }^{1}$ Department of Cardiology, Istanbul School of Medicine, Istanbul University, Istanbul, Turkey \\ ${ }^{2}$ Department of Oncology, Istanbul School of Medicine, Istanbul University, Istanbul, Turkey
}

\begin{abstract}
Background: The use of antracycline (ANT) in breast cancer has been associated with adverse cardiac events. Two-dimensional (2D) strain imaging (SI) can provide a more sensitive measure of altered left ventricular (LV) systolic function. We aimed to evaluate the preventive effect of carvedilol administration assessed by SI in a patient with breast cancer treated with ANT.

Methods: Patients receiving ANT were randomly assigned to the carvedilol- or placebo-receiving group. Each received an echocardiographic examination with conventional $2 D$ echocardiography, pulsed tissue Doppler, and 2D SI prior to and 6 months post ANT treatment. Results: During the 6-month follow-up period there were no patient deaths or interrupted chemotherapy treatments due to doxorubicin-induced cardiotoxicity. Both left ventricular ejection fraction (LVEF) and fractional shortening (FS) were within normal limits for all patients before and after ANT therapy. EF, FS and LV dimensions were measured using M-mode echocardiography and found to be similar in both groups before and after ANT therapy. The mean $E F, F S$, and LV echocardiograph baseline and control dimensions were similar in both groups after 6 months. Though baseline SI parameters were similar between the groups, there was a significant decrease in LV basal septal and basal lateral peak systolic strain in the control group compared to the carvedilol group.
\end{abstract}

Conclusions: These results indicate that carvedilol has a protective effect against the cardiotoxicity induced by ANT. (Cardiol J 2014; 21, 5: 509-515)

Key words: strain imaging, antracycline induced cardiotoxicity, echocardiography, carvedilol, beta-blocker

\section{Introduction}

Worldwide, breast cancer is a major public health problem with a lifetime risk of 1 in 10 and a mortality of approximately 1 in 33 [1]. Antracyclines (ANT) are potent, broad-spectrum antineoplastic agents used widely in the treatment of breast cancer. Their introduction has led to improved

Address for correspondence: Fahrettin Oz, MD, Department of Cardiology, Istanbul School of Medicine, Istanbul University, Istanbul Millet Street, Capa 34390, Istanbul, Turkey, tel: 90212414 20 00/31422, fax: 902125340768 , e-mail: fahrettin_oz@hotmail.com

Received: 26.08.2013 Accepted: 16.09.2013 
survival rates, but their therapeutic success is limited by potentially life-threatening cardiotoxicity [2-4]. Although cardiotoxicity is a well-known side effect of ANT, detection of subclinical myocardial contractility impairment at a latent stage is difficult. The adopted diagnostic approach to detect cardiac damage is based primarily upon the estimation of left ventricular (LV) ejection fraction (EF) or fractional shortening (FS). Preclinical identification of LV dysfunction is crucial, as early therapy will allow complete recovery. M-mode echocardiography imaging allows the assessment of muscle deformation, but has limited value in determining the number of myocardial segments since it allows deformation measurement in only the radial direction. Thus, it has been suggested that LV contractile function assessment be conducted using standard M-mode echocardiography systolic performance overestimates [5]. However, strain rate imaging can be used to quantify local myocardial deformation at a high temporal resolution. Recent studies have demonstrated the utility of strain imaging (SI) for the early detection of ANT-induced cardiomyopathy prior to alterations in conventional LVEF.

The mechanisms of ANT-induced cardiotoxicity (AIC) include the formation of free reactive oxygen radicals, direct DNA damage, apoptosis, the activation of immune reactions in the heart, changes in calcium transport, histamine release, and coagulation system activation leading to coronary artery thrombosis [6]. Carvedilol is a nonselective, adrenergic blocker which also has antioxidant, anti-apoptotic and vasodilating properties. Considering its antioxidant and anti-apoptotic effects, carvedilol may also be beneficial for the prevention of AIC, yet few clinical trials of the prophylactic role of carvedilol on AIC have been conducted $[7,8]$. In this study, we evaluated the preventive effect of carvedilol using strain imaging in patients with breast cancer treated with ANT.

\section{Methods}

This prospective and randomized study consisted of 80 female patients with breast cancer and anticipated ANT therapy, consecutively admitted to the Medical Oncology Department, Institute of Oncology, Istanbul University between 2012 and 2013. Exclusion criteria included previous chemotherapy or radiotherapy; cardioprotective drug use, such as angiotensin-converting enzyme inhibitors, angiotensin receptor blockers, calcium channel blockers, statins, aldosterone receptor antagonist, and other beta-blockers; the presence of any cardiac disease detected upon baseline evaluation and echocardiography; and a history of chronic disease, such as diabetes, hypertension, chronic renal or hepatic failure, and any contraindication to carvedilol use. Following study enrollment, participants underwent a cardiac evaluation, a standard electrocardiogram (ECG), a conventional echocardiography and SI. Eligible patients were randomly assigned to either the carvedilol or control group according to a computer-generated sequence. The carvedilol group was administered a dose of $12.5-\mathrm{mg}$ oral carvedilol prior to computed tomography, and followed by an oral carvedilol maintenance dose of $12.5 \mathrm{mg}$ daily for 6 months during chemotherapy. The control group did not receive any drug that could have cardioprotective effect.

The study was approved by the ethics committee of the Istanbul School of Medicine, Istanbul University. All patients provided written informed consent.

\section{Echocardiography}

Echocardiography was performed using the Vivid 7 echocardiography device (General Electrics, Milwaukee, WI, USA) tuned to a middle-range frequency $(3-8 \mathrm{MHz})$ broadband transducer. Diameter and EF were calculated according to the American Society of Echocardiography guidelines [9]. A pulsed-Doppler transmitral flow velocity profile was obtained from the apical 4-chamber view, and the sample volume was positioned immediately below the mitral valve leaflets. The following parameters were assessed: peak transmitral flow velocity in early diastole (peak E), peak transmitral flow velocity in late diastole (peak A), E/A ratio, isovolumic relaxation time, and isovolemic contraction time.

\section{Strain imaging}

Color-coded tissue Doppler data were acquired and stored as cine-loops. Both tissue velocity imaging (TVI) and SI measures were obtained from off-line analysis of stored loops by using custom software (Echopac, GE Systems, Oslo, Norway). Pulsed TVI analysis of the mitral annulus was performed in the apical 4-chamber view. A 5-mL sample volume was placed at the septal and lateral sites of the annulus. Settings were adjusted for a frame rate between $120 \mathrm{~Hz}$ and $180 \mathrm{~Hz}$, and a cineloop of 3 to 5 consecutive heart beats was recorded. TVI measures included peak systolic myocardial velocity, early and late diastolic myocardial velocities and their ratio at the basal segments of the LV lateral and septal walls. Care was taken to obtain an ultrasound beam parallel to the direction 
Table 1. Baseline characteristics and echocardiographic findings before anthracycline therapy.

\begin{tabular}{lccc}
\hline & Control group (n= 40) & Carvedilol group (n= 40) & P \\
\hline Age [years] & $52.9 \pm 11.2$ & $54.3 \pm 9.3$ & NS \\
Body mass index [kg/m ${ }^{2}$ ] & $22.9 \pm 2.4$ & $23.5 \pm 2.1$ & NS \\
Systolic blood pressure [mm Hg] & $121 \pm 26$ & $114 \pm 10$ & NS \\
Diastolic blood pressure [mm Hg] & $74 \pm 29$ & $74 \pm 10$ & NS \\
Hemoglobin [g/dL] & $13.8 \pm 3.2$ & $13.2 \pm 4.1$ & NS \\
Left atrium diameter [mm] & $32.3 \pm 5.1$ & $32.6 \pm 4.8$ & NS \\
Interventricular septum diameter [mm] & $10.3 \pm 1.3$ & $10.2 \pm 1.1$ & NS \\
Posterior wall diameter [mm] & $9.4 \pm 1.2$ & $9.1 \pm 1.4$ & NS \\
Left ventricular diastolic size [mm] & $44.3 \pm 3.1$ & $45.1 \pm 4.2$ & NS \\
Left ventricular systolic size [mm] & $27.1 \pm 6.1$ & $28.3 \pm 4.3$ & NS \\
Ejection fraction & $65 \pm 4.5$ & $66 \pm 6.1$ & NS \\
Fractional shortening & $40.6 \pm 5.1$ & $41.1 \pm 4.9$ & NS \\
E [m/s] & $79.4 \pm 18$ & $76 \pm 14$ & NS \\
A & $68 \pm 19$ & $65 \pm 11$ & NS \\
Isovolumic relaxation time & $65.3 \pm 18.9$ & $63.4 \pm 17.2$ & NS \\
E/A & $1.1 \pm 0.3$ & $1.18 \pm 0.4$ & NS \\
Septal S [mm] & $7.1 \pm 1.2$ & $7.2 \pm 1.3$ & NS \\
Lateral S [mm] & $8.8 \pm 2.1$ & $9.1 \pm 1.7$ & NS \\
Septal SSR [s ${ }^{-1}$ ] & $0.94 \pm 0.2$ & $0.92 \pm 0.14$ & NS \\
Lateral SSR [s ${ }^{-1}$ ] & $1.1 \pm 0.18$ & $1.04 \pm 0.21$ & NS \\
Septal SS [\%] & $19.3 \pm 4.1$ & $20.2 \pm 3.2$ & NS \\
Lateral SS [\%] & $17.3 \pm 3.2$ & $17.8 \pm 5.1$ & NS \\
\hline
\end{tabular}

SSR — systolic strain rate; SS — peak systolic strain; NS — non significant

of the mitral annulus motion. SI measures included peak systolic strain and strain rate, peak early and late diastolic strain rate at the basal segments of the LV lateral and septal walls, as SI is angle-dependent and longitudinal velocities are highest in the basal LV segments and diminish toward the apex. For all strain parameter measurements, the sample volume, oval and $6 \times 12 \mathrm{~mm}$ in size, was placed in the basal inner half of the LV myocardium at the septum and the lateral wall to keep the angle between the Doppler beam and the endocardium smaller than $30^{\circ}$. The narrowest possible image sector angle was used to achieve the maximum color Doppler frame rate. The frame rates ranged 154-184 fps. Strain rate was expressed as $1 / \mathrm{s}$. The strain data were expressed as negative percentage values and measured from systolic strain curves. All TVI and SI measurements were calculated from 3 consecutive cycles and the average of 3 measurements was recorded. The intra-observer variability for strain was $3.7 \pm 4.2 \%$.

\section{Statistical analysis}

Statistical analysis was performed with SPSS 17.0 for Windows (SPSS, Chicago, IL, USA). Con- tinuous data are expressed as means \pm standard deviation, and categorical data as percentages. Student's t-test was performed to compare unpaired samples. Differences between groups before and after therapy were compared with a paired t-test. The results are presented as relative risks (RR) with $95 \%$ confidence intervals (CI). A value of $p<0.05$ was considered to indicate statistical significance. The power analysis revealed that with this sample size ( $n=40$ vs. $n=40)$, the power level of this study was more than 0.90 for all SI parameters.

\section{Results}

Baseline characteristics are shown in Table 1. There were no significant differences between the groups in terms of age, body mass index, hemoglobin levels, systolic and diastolic pressure. The cumulative ANT chemotherapy doses were similar between the groups $\left(523.3 \mathrm{mg} / \mathrm{m}^{2}\right.$ in the control group, $535.6 \mathrm{mg} / \mathrm{m}^{2}$ in the carvedilol group). No patients died or interrupted chemotherapy due to doxorubicin-induced cardiotoxicity during the follow-up period. 
Table 2. M-mode echocardiographic and strain imaging findings after anthracycline therapy.

\begin{tabular}{|c|c|c|c|}
\hline & Control group $(n=40)$ & Carvedilol group $(n=40)$ & $\mathbf{P}$ \\
\hline Left atrium diameter [mm] & $34.3 \pm 6.1$ & $33.4 \pm 5.8$ & NS \\
\hline Interventricular septum diameter [mm] & $10.1 \pm 1.2$ & $10.3 \pm 0.9$ & NS \\
\hline Posterior wall diameter [mm] & $9.3 \pm 1.1$ & $9.2 \pm 1.5$ & NS \\
\hline Left ventricular diastolic size [mm] & $44.1 \pm 4.1$ & $44.6 \pm 3.2$ & NS \\
\hline Left ventricular systolic size [mm] & $27.6 \pm .5 .1$ & $28.1 \pm 4.3$ & NS \\
\hline Ejection fraction & $63.3 \pm 4.8$ & $64.1 \pm 5.1$ & NS \\
\hline Fractional shortening & $40.8 \pm 5$ & $40.1 \pm 4.5$ & NS \\
\hline$E[\mathrm{~m} / \mathrm{s}]$ & $78.8 \pm 18.1$ & $75.3 \pm 14.2$ & NS \\
\hline A & $69 \pm 16$ & $65.3 \pm 11$ & NS \\
\hline Isovolumic relaxation time & $71.3 \pm 16.8$ & $61.4 \pm 17.2$ & 0.01 \\
\hline$E / A$ & $1.09 \pm 0.4$ & $1.17 \pm 0.3$ & NS \\
\hline Septal S & $6.9 \pm 1$ & $7.2 \pm 0.9$ & NS \\
\hline Lateral S & $8.6 \pm 1.8$ & $9 \pm 2.1$ & NS \\
\hline Septal SSR $\left[\mathrm{s}^{-1}\right]$ & $0.7 \pm 0.14$ & $0.94 \pm 0.22$ & $<0.005$ \\
\hline Lateral SSR $\left[\mathrm{s}^{-1}\right]$ & $0.72 \pm 0.2$ & $1.08 \pm 0.26$ & $<0.005$ \\
\hline Septal SS [\%] & $16 \pm 4.3$ & $20.1 \pm 5.3$ & $<0.005$ \\
\hline Lateral SS [\%] & $14 \pm 6.1$ & $18.2 \pm 5.6$ & $<0.005$ \\
\hline
\end{tabular}

SSR — systolic strain rate; SS — peak systolic strain; NS — non significant

Table 3. Findings before and after anthracycline therapy in all study patients.

\begin{tabular}{|c|c|c|c|}
\hline & Before ANT therapy $(n=80)$ & After ANT therapy $(n=80)$ & $\mathbf{P}$ \\
\hline Left atrium diameter [mm] & $32.4 \pm 4.9$ & $33.8 \pm 5.9$ & NS \\
\hline Interventricular septum diameter [mm] & $10.2 \pm 1.2$ & $10.2 \pm 1.1$ & NS \\
\hline Posterior wall diameter [mm] & $9.3 \pm 1.3$ & $9.2 \pm 1.3$ & NS \\
\hline Left ventricular diastolic size $[\mathrm{mm}]$ & $44.7 \pm 3.7$ & $44.3 \pm 3.6$ & NS \\
\hline Left ventricular systolic size [mm] & $27.6 \pm 5.1$ & $27.8 \pm 4.7$ & NS \\
\hline Ejection fraction & $65.5 \pm 5.1$ & $63.8 \pm 5$ & NS \\
\hline Fractional shortening & $40.8 \pm 5.1$ & $40.4 \pm 4.7$ & NS \\
\hline $\mathrm{E}[\mathrm{m} / \mathrm{s}]$ & $77.7 \pm 16.1$ & $76.7 \pm 17.1$ & NS \\
\hline A & $66.3 \pm 15$ & $66.7 \pm 13$ & NS \\
\hline Isovolumic relaxation time & $64.1 \pm 17.9$ & $66.1 \pm 17.8$ & NS \\
\hline$E / A$ & $1.4 \pm 0.3$ & $1.3 \pm 0.3$ & NS \\
\hline Septal S & $7.15 \pm 1.2$ & $7.08 \pm 1$ & NS \\
\hline Lateral S & $8.9 \pm 1.9$ & $8.8 \pm 2$ & NS \\
\hline Septal SSR $\left[\mathrm{s}^{-1}\right]$ & $0.93 \pm 0.17$ & $0.81 \pm 0.18$ & 0.008 \\
\hline Lateral SSR $\left[\mathrm{s}^{-1}\right]$ & $1.07 \pm 0.19$ & $0.87 \pm 0.2$ & $<0.005$ \\
\hline Septal SS [\%] & $19.7 \pm 3.7$ & $18 \pm 4.7$ & 0.03 \\
\hline Lateral SS [\%] & $17.6 \pm 4.1$ & $16.1 \pm 5.8$ & 0.05 \\
\hline
\end{tabular}

SSR - systolic strain rate; SS - peak systolic strain; NS — non significant

\section{Echocardiography}

LVEF and FS were within normal limits in all patients after ANT therapy. EF, FS and LV dimensions measured by M-mode echocardiography were similar in all groups following ANT therapy (Table 2). Additionally, the mean EF, FS, and LV dimensions of both groups were similar at baseline and control echocardiography after 6 months (Table 3). The diastolic function parameters showed no significant differences between groups following ANT therapy, with the exception of isovolumic relaxation time (Table 2). 


\section{Strain imaging}

Strain imaging parameters were comparable between groups at baseline, and were within normal limits (Table 1). The septal and lateral systolic strain and strain rate values, which demonstrate longitudinal myocardial function, were significantly decreased in control patients compared to the carvedilol group following 6 months of ANT therapy (Table 2). In addition, the septal and lateral systolic strain and strain rate values were decreased significantly in control echocardiography compared to baseline echocardiography (Table 3).

\section{Discussion}

This randomized, controlled study evaluated the protective role of carvedilol in the development of silent AIC in patients with breast cancer. With a follow-up time of 6 months, it was possible to detect the early side-effects of AIC. Our major finding was that SI was significantly decreased in the control group compared to the carvedilol group following ANT therapy. However, the echocardiographic parameters identifying $L V$ function were not different between the groups. Interpretations of our findings have included the fact that ANT-cardiomyopathy (CMP) may have a regional pattern; thus the function of some myocardial segments may compensate for dysfunctional segments leading to a preserved LVEF in the early stages of cardiotoxicity. Another possible contributing factor to the higher sensitivity of strain compared to the LVEF may be the difference in inter-observer variability.

Echocardiography is one of the most widely used noninvasive methods for the early detection and monitoring of ANT-induced CMP [10]. Early detection is of paramount importance as it allows for the use of cardioprotective agents. Longitudinal myocardial function may be impaired while systolic function remains normal. Standard gray-scale imaging not only allows the assessment of cardiac structure, but also provides information on myocardial function. Standard echocardiographic measurements, such as endocardial FS and EF, reflect the chamber dynamics and are not direct measurements of longitudinal function. As many diseases result in the regional dysfunction of the ventricle, ultrasonograhic assessment of regional myocardial dysfunction remains an important goal in clinical cardiology $[11,12]$. Recent studies showed that strain- and strain rate-imaging were more sensitive indices in identifying ANT-induced myocardial systolic changes than standard echocardiographic parameters [13, 14]. Stoodley et al. [15] revealed that while greater than $10 \%$ reductions in longitudinal and radial peak LV strain measurements were observed following ANT therapy in half of the participants, a corresponding reduction in $\mathrm{EF}$ of greater than $10 \%$ was not observed in any of the participants. Further, Sawaya et al. [16] recently reported that reduced longitudinal and radial strain following 3 months of cancer treatment could predict the development of cardiotoxicity, while reduced $\mathrm{EF}$ following 3 months could not.

Several hypotheses have been advanced to explain AIC. Most involve the generation of reactive oxygen species, which induce apoptosis and cardiac myocyte damage [17]. Other mechanisms implicated in the pathogenesis AIC include lipid peroxidation, gene expression reduction, nucleic acid and protein synthesis inhibition, adrenergic function alteration and disruption of mitochondrial $\mathrm{Ca}^{2+}$ homeostasis [18-20]. ANT side effects are cumulatively dose dependent and irreversible. The risk of developing ANT-induced CMP is affected by many factors including age, concomitant use of other chemotherapeutics and targeted therapy agents (e.g., trastuzumab), prior irradiation exposure, underlying heart disease and high cumulative doses [21]. In addition, the incidence of ANT-induced CMP largely depends on the type of chemotherapeutic agent being used (e.g., epirubicin and liposomal doxorubucin are less cardiotoxic than adriamycin). Several methods, including pharmacokinetic modification by liposomal encapsulation, alteration of chemical structure leading to drugs such as epirubicin, altering drug infusion, and attenuation of iron chelation through pretreatment with dexrazoxane have been associated with a reduction in cardiovascular events in ANT treated patients; however most of these strategies are not in common practice in the clinical setting [22]. Beta-blockers have been considered agents for the treatment of systolic heart failure [23]. Beneficial effects of carvedilol on morbidity and all-cause mortality have been demonstrated in heart failure patients [24]. Few reports are available on the cardioprotective use of beta-blockers in patients receiving ANT $[8,25]$. Trials conducted among limited number of patients have shown that co-administration of carvedilol during ANT therapy could prevent ANT-induced CMP. Additionally, Jonnson et al. [26] showed that carvedilol does not reduce the effectiveness of anti-neoplastics, which is an important consideration if it is to be administered together with ANT. The mechanisms behind the protective effect of carvedilol against ANT-induced CMP were not fully explained, but 
it is assumed to be due to the antioxidant action of carvedilol and its metabolites [27]. Other possible mechanisms include: restoration of the activity of the sarcoplasmic reticulum Ca-ATPase (SERCA2) in myocytes, the expression inhibition of the SERCA2 suppressor gene [28, 29]. Additionally, carvedilol treatment is associated with the inhibition of the apoptotic signaling pathway [8]. However, at present, there is no phase III trial data that strongly encourages the routine prophylactic administration of carvedilol for protection against ANT-induced CMP.

In light of the above mentioned facts, the protective effect of carvedilol could be detected more sensitively by SI. The present study showed that co-administration of carvedilol during ANT therapy preserves several subtle abnormalities in myocardial function that were detected in the asymptomatic period and normal EF, as identified by SI. The results of our study indicate that carvedilol treatment has some protective effects againist AIC in the short-term but we can not extrapolate our results to the long-term because of the short follow-up of our study. While EF and FS were similar in both group echocardiographies, septal and lateral systolic strain and strain rate values were significantly lower in control patients compared to the carvedilol group. The significant reduction in SI parameters was in agreement with the results of ANT-induced therapy in children described in Ganame et al. [30]. In addition there were no significant differences in diastolic function measured after ANT therapy in our patients. Similar results were reported by Ewer et al. [31] and El-Shitany et al [25].

\section{Limitations of the study}

Our study has several limitations; including its open label design, and the relatively small sample size. However the detection of subtle changes in myocardium of breast cancer patients exposed to ANT with SI and reversal of these deleterious effects with carvedilol is a promising finding for future therapeutical interventions.

\section{Conclusions}

The preventative effect of carvedilol on longitudinal LV systolic function during ANT therapy may be documented by strain rate imaging, which is afterload independent at a time when the other parameters obtained from standard M-mode echocardiographic analysis remain normal. Our results will require long-term follow-up evaluations for future cardiovascular events in the carvedilol patient group.

\section{Conflict of interest: None declared}

\section{References}

1. WHO Disease and injury country estimates. World Health Organization. 2009. Retrieved Nov. 11, 2009.

2. Mornoş C, Petrescu L. Early detection of anthracycline-mediated cardiotoxicity: the value of considering both global longitudinal left ventricular strain and twist. Can J Physiol Pharmacol, 2013; 91: 601-607.

3. Von Hoff DD, Layard MW, Basa P et al. Risk factors for doxorubicin-induced congestive heart failure. Ann Intern Med, 1979; 91: 710-717.

4. Swain SM, Whaley FS, Ewer MS. Congestive heart failure in patients treated with doxorubicin: A retrospective analysis of three trials. Cancer, 2003; 97: 2869-2879.

5. Heimdal A, Stoylen A, Torp $\mathrm{H}$ et al. Real-time strain rate imaging of the left ventricle by ultrasound. J Am Soc Echocardio, 1998; 11: 1013-1019.

6. Singal PK, Li T, Kumar D, Danelisen I, Iliskovic N. Adriamycin-induced heart failure: mechanism and modulation. Mol Cell Biochem, 2000; 207: 77-86.

7. Dulin B, Abraham WT. Pharmacology of carvedilol. Am J Cardiol, 200; 93: 3B-6B.

8. Kalay N, Basar E, Ozdogru I et al. Protective effects of carvedilol against anthracycline-induced cardiomyopathy. J Am Coll Cardiol, 2006; 48: 2258-2262.

9. Sahn DJ, DeMaria A, Kisslo J et al. Recommendations regarding quantitation in M-mode echocardiography: Results of a survey of echocardiographic measurements. Circulation, 1978; 58: 1072-1083.

10. Steinherz LJ, Graham T, Hurwitz R et al. Guidelines for monitoring of anthracycline cardiomyopathy: A rebuttal. Pediatrics, 1994; 94: 782-784.

11. D'Andrea A, Stisi S, Bellissimo S et al. Early impairment of myocardialfunction in systemic sclerosis:non-invasiveassessment by Doppler myocardial and strain rate imaging. Eur J Echocardiogr, 2005; 6: 407-418.

12. Hamdy AM. Use of strain and tissue velocity imaging for early detection of regional myocardial dysfunction in patients with beta thalassemia. Eur J Echocardiogr, 2007; 8: 102-109.

13. Jurcut R, Wildiers H, Ganame J et al. Strain rate imaging detects early cardiac effects of pegylated liposomal doxorubicin as adjuvant therapy in elderly patients with breast cancer. J Am Soc Echocardiogr, 2008; 21: 1283-1289.

14. Cheung YF, Hong WJ, Chan GC, Wong SJ, Ha SY. Left ventricular myocardial deformation and mechanical dyssynchrony in children with normal ventricular shortening fraction after anthracycline therapy. Heart, 2010; 96: 1137-1141.

15. Stoodley PW, Richards DA, Hui R et al. Two-dimensional myocardial strain imaging detects changes in left ventricular systolic function immediately after anthracycline chemotherapy. Eur J Echocardiogr, 2011; 12: 945-952.

16. Sawaya H, Sebag IA, Plana JC et al. Early detection and prediction of cardiotoxicity in chemotherapy-treated patients. Am J Cardiol, 2011; 107: 1375-1380.

17. Demant EJ. Inactivation of cytochrome c oxidase activity in mitochondrial membranes during redox cycling of doxorubicin. Biochem Pharmacol, 1991; 41: 543-552. 
18. Palmeira CM, Serrano J, Kuehl DW, Wallace KB. Preferential oxidation of cardiac mitochondrial DNA following acute intoxication with doxorubicin. Biochim Biophys Acta, 1997; 1321: 101-106.

19. Mimnaugh EG, Trush MA, Bhatnagar M, Gram TE. Enhancement of reactive oxygen-dependent mitochondrial membrane lipid peroxidation by the anticancer drug adriamycin. Biochem Pharmacol, 1985; 34: 847-856.

20. Kang YJ, Chen Y, Yu A, Voss-McCowan M, Epstein PN. Overexpression of metallothionein in the heart of transgenic mice suppresses doxorubicin cardiotoxicity. J Clin Invest, 1997; 100: 1501-1506.

21. Pereira OG, Alegria JP, Gouveia AP. Índice Nacional Terapêutico - Edição de Mesa. Tupam Editores, Lisboa, Portugal 2005: 633-856, 1429-1513.

22. Suter TM, Ewer MS. Cancer drugs and then heart: Importance and management. Eur Heart J, 2013; 34: 1102-1111.

23. Tenero D, Boike S, Boyle D et al. Steady-state pharmacokinetics of carvedilol and its enantiomers in patientswith congestive heart failure. J Clin Pharmacol, 2000; 40: 844-853.

24. Packer M, Bristow MR, Cohn JN et al. for the U.S. Carvedilol Heart Failure Study Group. The effect of carvedilol on morbidity and mortality in patients with chronic heart failure. $\mathrm{N}$ Engl J Med, 1996; 334: 1349-1355.
25. El-Shitany NA, Tolba OA, El-Shanshory MR, El-Hawary EE. Protective effect of carvedilol on adriamycin-induced left ventricular dysfunction in children with acute lymphoblastic leukemia. J Card Fail, 2012; 18: 607-613.

26. Jonsson O, Behnam-Motlagh P, Persson M, Henriksson R, Grankvist K. Increase in doxorubicin cytotoxicity by carvedilol inhibition of P-glycoprotein activity. Biochem Pharmacol, 1999; 58: 1801-1806.

27. Oliveira PJ, Bjork JA, Santos MS et al. Carvedilol-mediated antioxidant protection against doxorubicin-induced cardiac mitochondrial toxicity. Toxicol Appl Pharmacol, 2004; 200: 159- 168.

28. Matsui H, Morishima I, Numaguchi Y, Toki Y, Okumura K, Hayakawa T. Protective effects of carvedilol against doxorubicininduced cardiomyopathy in rats. Life Sci, 1999; 65: 1265-1274.

29. Jonsson O, Behnam-Motlagh P, Persson M, Henriksson R, Grankvist K. Increase in doxorubicin cytotoxicity by carvedilol inhibition of P-glycoprotein activity. Biochem Pharmacol, 1999; 58: 1801-1806.

30. Ganame J, Claus P, Uyttebroeck A et al. Myocardial dysfunction late after low-dose anthracycline treatment in asymptomatic pediatric patients. J Am Soc Echocardiogr, 2007; 20: 1351-1358.

31. Ewer MS, Ali MK, Gibbs HR et al. Cardiac diastolic function in pediatric patients receiving doxorubicin. Acta Oncol, 1994; 33: 645-649. 